\title{
Resgate fisioterapêutico para pacientes com comprometimento da função pulmonar e câncer de pulmão
}

\author{
Alessandra K. Peres, ${ }^{1 \star}$ Ana C. de A. Carvalho, ${ }^{2}$ Márcia dos G. Peixoto, ${ }^{1}$ Eduardo H. Saito ${ }^{3}$
}

\section{Resumo}

Frente à rotina de estadiamento dos pacientes com neoplasia pulmonar admitidos na enfermaria de cirurgia torácica do Hospital Universitário Pedro Ernesto, frequentemente nos deparamos com casos que possuem indicação de abordagem cirúrgica, porém apresentam prova de função pulmonar evidenciando valores de volume expiratório forçado no primeiro segundo abaixo dos limites de referência admissíveis, contraindicando essa intervenção face ao risco aumentado de morbimortalidade perioperatória. Para que pacientes inelegíveis às cirurgias de ressecção pulmonar possam ter a chance de serem submetidos a esse tipo de intervenção curativa, tem sido investigada a eficácia da intervenção fisioterapêutica na otimização da função pulmonar por meio de exercícios e ventilação não invasiva, no período pré-operatório. Este relato de caso trata da abordagem fisioterapêutica, em uma paciente previamente inelegível ao procedimento proposto, associando ventilação não invasiva a exercícios resistidos de membros superiores. Após duas semanas de tratamento, verificou-se melhora dos valores espirométricos, tornando-a elegível à intervenção cirúrgica.

Descritores: Neoplasias pulmonares; Cirurgia torácica; Reabilitação.

\section{Abstract \\ Physical therapy for patients with lung cancer and compromised pulmonary function}

In the staging of in-patients with pulmonary neoplasms in the thoracic surgery infirmary of Hospital Universitário Pedro Ernesto we often face cases in which surgery is advised, but the pulmonary function test show a forced expiratory volume in one second (FEV1) below acceptable reference values, indicating such surgical intervention to be inadvisable due to high periprocedural morbimortality. In order to make such patients eligible to pulmonary resection, the efficacy of physical therapy to improve pulmonary function through preprocedural exercise and non-invasive ventilation has been investigated. This case report presents the physical therapy of a patient previously ineligible for a proposed procedure: non-invasive ventilation associated with resisted exercise for the upper limbs, which made her eligible for surgical intervention.

Keywords: Lung neoplasms; Thoracic surgery; Rehabilitation.
1. Serviço de Fisioterapia. Hospital Universitário Pedro Ernesto. Universidade do Estado do Rio de Janeiro. Rio de Janeiro, RJ, Brasil.

2. Faculdade de Fisioterapia. Instituto Federal de Educação, Ciência e Tecnologia do Rio de Janeiro. Rio de Janeiro, RJ, Brasil.

3. Serviço de Cirurgia Torácica. Hospital Universitário Pedro Ernesto. Universidade do Estado do Rio de Janeiro. Rio de Janeiro, RJ, Brasil.

*Endereço para correspondência:

Serviço de Fisioterapia, HUPE, UERJ

Boulevard 28 de Setembro, 77

Rio de Janeiro, RJ, Brasil. CEP: 20551-030.

E-mail: alessandrakrykhtine@gmail.com

Revista HUPE, Rio de Janeiro, 2015;14(Supl. 1):97-100

doi: 10.12957/rhupe.2015.17771

Recebido em 03/06/2015. Aprovado em 15/07/2015.

\section{Resumen}

Recuperación terapéutica para pacientes con compromiso de la función pulmonar y cáncer de pulmón

Recuperación terapéutica para pacientes con compromiso de la función pulmonar y cáncer de pulmón pulmonary resection, the efficacy of physical therapy to improve pulmonary function through preprocedural exercise and non-invasive ventilation has been investigated. This case report presents the physical therapy of a patient previously ineligible for a proposed procedure: non-invasive ventilation associated with resisted exercise for the upper limbs, which made her eligible for surgical intervention.

Palabras clave: Neoplasias pulmonares; Cirugía torácica; Rehabilitacíon. 


\section{Caso clínico}

\section{Introdução}

O câncer de pulmão é um dos tipos de câncer mais frequentes em todo o mundo e, segundo a estimativa brasileira do ano 2014, corresponde a valores de risco estimados de 16,79 casos novos a cada 100 mil homens e 10,75 a cada 100 mil mulheres. ${ }^{1}$

A ressecção pulmonar é o principal método de escolha para tratamento em pacientes com câncer de pulmão em estágios iniciais, apesar de haver risco de morbidade e mortalidade no período pós-operatório. Com o objetivo de avaliar o risco perioperatório do tratamento curativo, é realizada a espirometria, como prova de função pulmonar, para orientação entre os manejos conservador e cirúrgico. Na literatura, é estabelecido que o volume expiratório forçado no primeiro segundo $\left(\mathrm{VEF}_{1}\right)$ em litros, assim como seu valor predito, em porcentagem, deve ser $1,5 \mathrm{~L}-60 \%$ e 2,0 L - 80\%, para a realização segura de lobectomias e pneumectomias, respectivamente. 23,4

Para que pacientes inelegíveis a cirurgias de ressecção pulmonar possam ter a chance de serem submetidos a esse tipo de intervenção curativa, tem sido demonstrado que a otimização da função cardiopulmonar pela intervenção fisioterapêutica com utilização de exercícios e também de ventilação não invasiva no período pré-operatório é factível. $5,6,7,8,8,10$

Nosso objetivo, neste relato de caso, foi defender o programa fisioterapêutico como uma importante estratégia para melhorar a função pulmonar de um paciente, tornando-o elegível para a ressecção cirúrgica.

\section{Paciente}

Paciente O. A. G. E. S., sexo feminino, 73 anos, natural de Maricá - RJ, aposentada, trabalhava como empregada doméstica e era residente do Rio de Janeiro - RJ.

Na admissão, as queixas principais foram: tosse seca e dor pleurítica com duração de três anos, incluindo história patológica prévia de hipertensão arterial sistêmica e câncer colorretal tratado com colectomia total abdominal. Ex-tabagista de 100 maços/ano, com interrupção recente.

Na radiografia de tórax, em incidência posteroanterior, evidenciou massa pulmonar em lobo inferior direito, sugerindo lesão pulmonar.

A tomografia computadorizada de tórax anual para acompanhamento de nódulo pulmonar evidenciou massa de lobo inferior direito, histologicamente identificada como carcinoma de células escamosas, após biópsia transbrônquica. Estadiamento da neoplasia pulmonar T3 N2 M0, classificação IIIA.

Apresentou ecocardiografia transtorácica com fração de ejeção 60\%; disfunção diastólica tipo I, função sistólica global e segmentar de ventrículo esquerdo preservadas.

Medicamentos em uso: AAS $100 \mathrm{mg} /$ dia; losartana potássica $50 \mathrm{mg} /$ dia; sinvastatina $20 \mathrm{mg} /$ dia; carbonato de cálcio $500 \mathrm{mg} /$ dia; vitamina D 5000U/dia; alendronato de sódio $70 \mathrm{mg} / \mathrm{semana}$; clonazepam 0,5 mg/dia; nebulização com brometo de ipratrópio $0,5 \mathrm{mg} /$ dia.

À avaliação fisioterapêutica apresentou padrão respiratório predominantemente costal; expansibilidade reduzida em hemitórax direito; ausculta pulmonar com murmúrio vesicular universalmente diminuído, sem ruídos adventícios. Deambulação com auxílio de órtese à direita. Os valores de força muscular respiratória, avaliados por meio da manovacuometria foram: pressão inspiratória máxima ( $\mathrm{PI}_{\text {máx }}$ ) $-42 \mathrm{cmH}_{2} \mathrm{O}$, pressão expiratória máxima $\left(\mathrm{PE}_{\text {máx }}\right)+38 \mathrm{cmH}_{2} \mathrm{O}$.

\section{Metodologia}

O programa de exercícios fisioterapêuticos proposto foi realizado pela paciente em duas semanas: dez sessões diárias de 30 minutos, sob ventilação não invasiva (VNI) a um fluxo de 7 1/min de oxigênio, com pressão positiva contínua nas vias aéreas (CPAP) $10 \mathrm{cmH}_{2} \mathrm{O}$, por meio de máscara facial.

As sessões eram iniciadas com alongamento dos principais músculos respiratórios torácicos por mobilização passiva em sequência. Foram realizados os exercícios resistidos em três séries de 15 repetições, de acordo com a descrição a seguir:

a) flexão e extensão da articulação glenoumeral, ativo-resistidas com halter de $1 \mathrm{~kg}-180^{\circ}$ plano sagital;

b) movimentos combinados na direção diagonal, cruzando a linha média (flexão, abdução e rotação externa; extensão, adução e rotação interna das articulações glenoumerais), ativos resistidos com halter de $1 \mathrm{~kg}$;

c) exercícios ativos resistidos, por meio de uma faixa elástica, para músculos torácicos. Flexão $\left(45^{\circ}\right)$ e extensão $\left(45^{\circ}\right)$ de articulação glenoumeral, com o antebraço fletido a $90^{\circ}$, em posição neutra.

Foram comparados os valores de capacidade vital forçada (CVF, CVF\%), $\mathrm{VEF}_{1}, \mathrm{VEF}_{1} \%, \mathrm{VEF}_{1} / \mathrm{CVF}, \mathrm{VEF}_{1} /$ $C V F \%, \mathrm{PI}_{\text {máx }}$ e $\mathrm{PE}_{\text {máx }}$, no período pré e pós-intervenção fisioterapêutica. 


\section{Resultados}

Houve aumento tanto de CVF em litros (1,78 - 2,21 L), quanto CVF\% (72,4\% - 89,8\%) e do $\mathrm{VEF}_{1}$ em litros $(1,21-1,49 \mathrm{~L})$, quanto $\mathrm{VEF}_{1} \%$ (65\% - 80,1\%), no período pré-intervenção fisioterapêutica, quando comparado aos obtidos após o tempo de tratamento (Figuras 1 e 2).

Osíndices $\mathrm{VEF}_{1} / \mathrm{CVF}_{\mathrm{VEF}} / \mathrm{CVF} \%$ também obtiveram mudança com o tratamento fisioterapêutico. $\mathrm{VEF}_{1} /$ CVF, no período pré e pós-intervenção foram de $68,18 \mathrm{e}$ 67,19 , respectivamente. A modificação da $\mathrm{VEF}_{1} / \mathrm{CVF} \%$ foi de $87,9 \%$ para $86,7 \%$ no mesmo intervalo de tempo.

Houve melhora também da força muscular respiratória, mensurada por meio da manovacuometria, quando comparados aos valores pré e pós-intervenção fisioterapêutica. $\mathrm{API}_{\text {máx }}$ evoluiu de $-42 \mathrm{cmH}_{2} \mathrm{O}$ para -72 $\mathrm{cmH}_{2} \mathrm{O}$, e a $\mathrm{PE}_{\text {máx }} 38 \mathrm{cmH}_{2} \mathrm{O}$ para $68 \mathrm{cmH}_{2} \mathrm{O}$ (Tabela 1).

\section{Discussão}

A função pulmonar limítrofe foi um fator de risco modificável no período pré-operatório em nosso relato de caso, já que houve aumento dos valores de $\mathrm{VEF}_{1}$. A reabilitação pulmonar realizada em duas semanas, incluindo treinamento de força muscular periférica associada à ventilação não invasiva, na modalidade pressão positiva contínua nas vias aéreas (CPAP), mostrou-se uma terapia capaz de alterar o prognóstico desta paciente.

Os resultados de Cesario e colaboradores, em 2007,6 corroboram com os apresentados em nosso relato.

Figura 1. Gráfico da capacidade vital forçada (CVF) em litros, e sua respectiva porcentagem, pré e pós-intercenção fisioterapêutica.

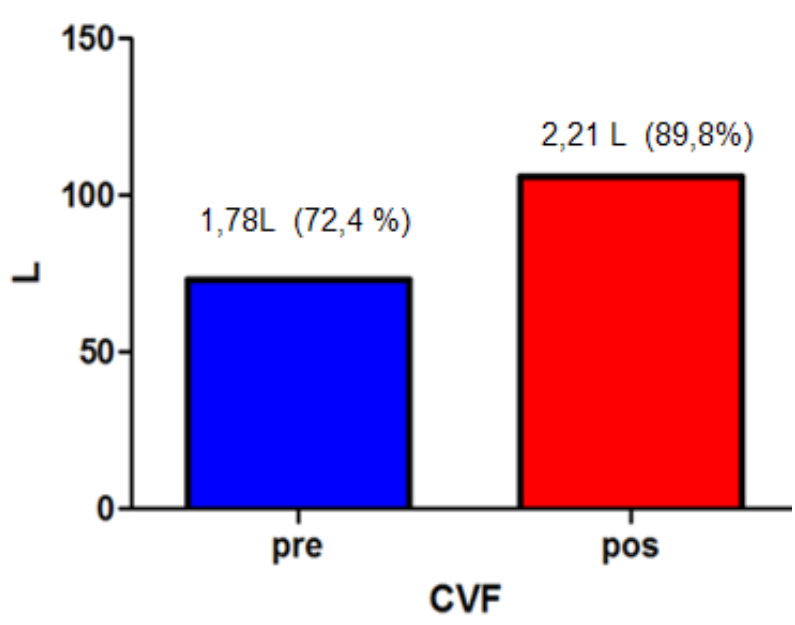

Nele, a reabilitação pulmonar, por meio de exercícios e estimulação elétrica funcional diafragmática, sem utilização VNI, foi aplicada em pacientes que foram contraindicados à cirurgia de ressecção pulmonar por câncer de pulmão por possuírem função pulmonar prejudicada. Houve diferença significativa na CVF e na porcentagem do $\mathrm{VEF}_{1}$ pós-intervenção.

Em relação à utilização de ventilação não invasiva, o estudo de Perrin e colaboradores ${ }^{10}$, em 2007, teve como objetivo observar a melhora da função pulmonar em pacientes com $\mathrm{VEF}_{1}$ menor que $70 \%$ do predito, indicados para lobectomia por câncer de pulmão. Foi comparado o grupo-intervenção, o qual realizou VNI no modo pressão de suporte nos períodos pré e pós-operatórios de maneira profilática, com grupo-controle, que recebeu tratamento convencional. No período pós-operatório, ambos os grupos obtiveram declínio significativo das variáveis espirométricas analisadas. Porém, os valores continuaram maiores no grupo-intervenção do que no grupo-controle.

Diaz e colaboradores 2002,1 $\mathrm{em}$ um estudo cego e randomizado com 36 pacientes portadores de doença pulmonar obstrutiva crônica (DPOC) estáveis, mostraram que a aplicação de ventilação não invasiva no modo CPAP durante três semanas, diminuiu significativamente os valores de CRF e volume residual (VR). Aumentos nos valores de $\mathrm{VEF}_{1}$ e CVF foram equivalentes, o que manteve a relação $\mathrm{VEF}_{1} / \mathrm{CVF}$ equilibrada e não aconteceu com nosso relato de caso, pois os valores de CVF aumentaram proporcionalmente mais do que os de $\mathrm{VEF}_{1}$, diminuindo essa relação na espirometria pós-

Figura 2. Gráfico do volume expiratório forçado no primeiro segundo $\left(\mathrm{VEF}_{1}\right)$ em litros e sua respectiva porcentagem, pré e pós-intervenção fisioterapêutica.

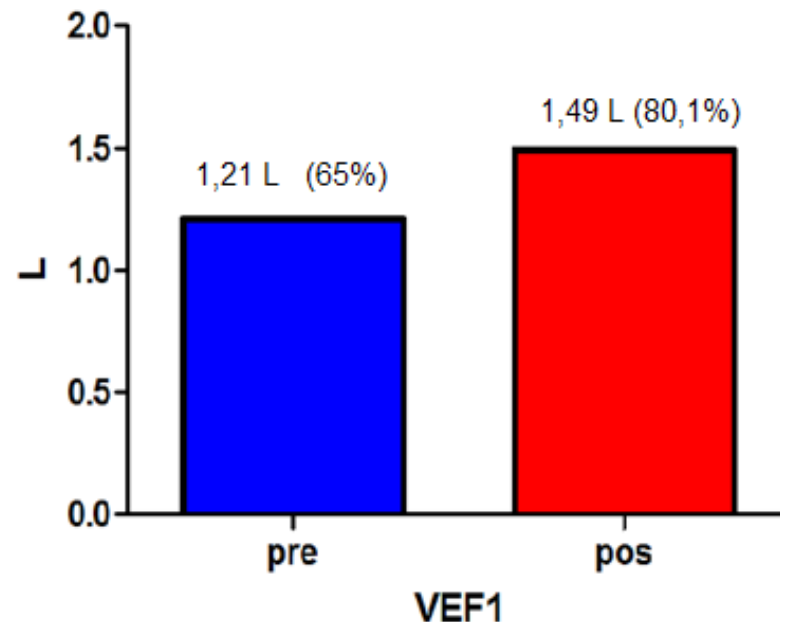




\section{Caso clínico}

-intervenção fisioterapêutica. Esses autores atribuíram as mudanças nos volumes pulmonares ao aumento do tempo expiratório, o que permitiu o esvaziamento de ar das unidades acometidas, as quais possuem maior constante de tempo. A redução da hiperinsuflação dinâmica também contribuiu para diminuir o esforço respiratório do paciente, fazendo com que este adotasse um novo padrão respiratório espontâneo, lento e profundo.

Tal fato pode justificar os achados de nosso estudo, já que nesse, a espirometria foi realizada duas semanas após o início dos atendimentos fisioterapêuticos, o que pode estar relacionado ao aprendizado de um novo padrão respiratório, favorecido pela diminuição da hiperinsuflação pulmonar pela aplicação da CPAP durante nossas intervenções terapêuticas.

$\mathrm{O}$ aumento da $\mathrm{PI}_{\text {máx }}$ pode ter contribuído para o aumento dos volumes pulmonares refletidos na prova de função pulmonar realizada após o período de intervenção.

\section{Conclusão}

A função pulmonar comprometida dessa paciente foi um fator de risco modificável, no período préoperatório, pois alguns valores espirométricos melhoraram após o programa fisioterapêutico. $\mathrm{O}$ fortalecimento da musculatura periférica, por meio de exercícios resistidos associados à ventilação não invasiva provavelmente foi o fator responsável pelo o aumento do $\mathrm{VEF}_{1}$ de $65 \%$ para $80,1 \%$, permitindo a resseção cirúrgica curativa em um paciente com prognóstico prévio limitado.

\section{Referências}

1. Estimativa 2014: incidência de câncer no Brasil / Instituto Nacional de Câncer José Alencar Gomes da Silva, Coordenação de Prevenção e Vigilância. Rio de Janeiro: INCA, 2014.

2. Kaza AK, Mitchell JD. Preoperative Pulmonary Evaluation of the Thoracic Surgical Patient. Thoracic Surgery Clinics. 2005;15:297-304.

3. Brunelli A, Rocco G. Spirometry: Predicting Risk and Outcome. Thoracic Surgery Clinics. 2008; 18:1-8.

4. Varela G, Novoa NM. Future Trends in Preoperative Evaluation. Thoracic Surgery Clinics. 2008; 18:31-37.

5. Brunelli A Charloux A, Bolliger CT, et al. ERS/ESTS clinical guidelines on fitness for radical therapy in lung cancer patients (surgery and chemo-radiotherapy).The European Respiratory Journal. 2009;34:17-41.

6. Cesario A, Ferri L, Galetta D, et al. Pre-operative pulmonary rehabilitation and surgery for lung cancer. Letter to the editor. Lung Cancer. 2007; 57:118-119.

7. Bobbio A, Chetta A, Ampollini L, et al. Preoperative pulmonary rehabilitation in patients undergoing lung resection for non-small cell lung cancer. European Journal of Cardiothoracic Surgery. 2008 jan;33(1):95-8.

8. Bobbio A, Chetta A, Internullo E, et al. Exercise capacity assessment in patients undergoing lung resection. European Journal of Cardiothoracic Surgery. 2009 mar;35(3):419-22.

9. Benzo R, Wigle D, Novotny P, et al. Preoperative pulmonary rehabilitation before lung cancer resection: Results from two randomized studies. Lung Cancer. 2011 Dec;74(3):441-5.

10. Perrin C, Jullien V, Vénissac N, et al. Prophilatic use of noninvasive ventilation in patients undergoing lung resectional surgery. Respiratory medicine. 2007;101:1572-1578.

11. Díaz O, Bégin P, Torrealba B, et al. Effects of noninvasive ventilation on lung hyperinflation in stable hypercapnic COPD. The European Respiratory Journal. 2002 Dec;20(6):1490-8. 